\title{
Therapeutic Angiogenesis of PLGA-Heparin Nanoparticle in Mouse Ischemic Limb
}

\author{
Lishan Lian, Feng Tang, Jing Yang, Changwei Liu, and Yongjun Li \\ Department of Vascular Surgery, Peking Union Medical College Hospital, Peking Union Medical College, \\ Chinese Academy of Medical Science, Beijing 100730, China \\ Correspondence should be addressed to Yongjun Li, yongj_93@hotmail.com
}

Received 11 January 2012; Revised 12 April 2012; Accepted 13 April 2012

Academic Editor: Haifeng Chen

Copyright ( 92012 Lishan Lian et al. This is an open access article distributed under the Creative Commons Attribution License, which permits unrestricted use, distribution, and reproduction in any medium, provided the original work is properly cited.

\begin{abstract}
Objective. To evaluate the possibility and efficacy of the nanoparticle encapsulating heparin as a novel delivery system to treat ischemic disease. Methods. Firstly, to synthesize the PLGA heparin and test the surface morphology, the average diameter, the loading efficiency, and the release time in vitro, then inject the PLGA heparin into mouse ischemic limbs to observe the perfusion recovery with LDPI at the time of postischemic 7, 14, 21, and 28 days, and, finally, test the expression of VEGF and HGF, the number of the neovessels and record the necrotic score of ischemic limbs. Results. The surface morphology of the PLGA heparin was smooth, the average diameter was $297 \mathrm{~nm}$, the loading efficiency was 5.35\%, and the release period maintained for 14 days. In animal experiment, the perfusion recovery, HGF expression level, and capillary density in PLGA-heparin group were significantly higher than that in control group, and this was consistent with less ischemic limb necrosis. Conclusion. Nanoparticle encapsulating heparin could be successful and efficient in ischemic disease. The therapeutic angiogenesis of PLGA heparin might be due to the prolongation of its biological effects with stimulating growth factor expression.
\end{abstract}

\section{Introduction}

Ischemic diseases are major cause of morbidity and mortality [1]. Though pharmacology, stents, and bypass have been advantageously developed in this area, some of the patients, with diabetes, hyperlipidemia, or hypertension, are poorer clinical outcomes as well as poorer responses to pharmacology and not eligible or poor candidates for revascularization [2]. There are currently no medical therapies available to improve perfusion and thereby alter the poor prognosis [3].

Angiogenesis is the growth and proliferation of new blood vessels from existing vascular structures [4]. Therapeutic angiogenesis seeks to employ this phenomenon for the treatment of disorders of inadequate tissue perfusion.

Currently, a lot of growth factors and stem cells have been tried as an angiogenic promoter. However, their clinical effects on patients are not as exciting as experimental results in animal models. Originally, heparin is a basic anticoagulant for the prevention and treatment of arterial and venous thromboembolic diseases [5-7]. The anticoagulant activity of heparin depends on a unique pentasaccharide which binds to antithrombin III (AT III), potentiates the inhibition of thrombin, and activates factor X (Xa) [8-10]. Heparin has also a number of other effects, including stimulating the expression of tissue factor pathway inhibitor, binding to numerous plasma and platelet proteins, acting with endothelial cells, and leukocytes, suppressing of platelet function, and improving vascular permeability. More importantly, heparin has been proved to improve angiogenesis in clinic [8]. However, heparin has pharmacokinetic and biological limitation of its short half-life. To maintain its function needs recurrent injection or continuous intravenous infusion.

Polymeric nanoparticles formulated from biodegradable copolymer, poly lactic-coglycolic acid (PLGA), have been extensively investigated in drug and gene delivery [11-14]. PLGA nanoparticles have advantages such as a controlled release of drug delivery, high stability, being easily uptaken into the cells by endocytosis, and targeting ability to specific tissue or organ by adsorption or coating with ligand materials at the surface of spheres. In addition, PLGA has good biocompatibility and has been approved by Food and Drug Administration for certain human clinical uses [1517]. 
In this study, we evaluated the possibility and efficiency of PLGA nanoparticles encapsulating unfractionated heparin as a novel drug delivery system to prolong heparin release and investigated the therapeutic angiogenesis efficacy in ischemic limbs of mice.

\section{Materials and Methods}

2.1. Preparation of PLGA-Heparin Nanoparticles. Double emulsion-solvent evaporation method was used to synthesize PLGA-heparin nanoparticles [18]. Briefly, $2 \mathrm{~mL}$ unfractionated heparin solution (12500 IU) was emulsified in $10 \mathrm{~mL}$ of PLGA solution ( $5 \% \mathrm{w} / \mathrm{v}$ in methylene chloride), using homogenizer for $3 \mathrm{~min}$ in ice bath. The water-in-oil emulsion was further emulsified in $50 \mathrm{~mL}$ of a $2 \%(\mathrm{w} / \mathrm{v})$ aqueous solution of polyvinyl alcohol (PVA, MW 30,000-70,000) for $3 \mathrm{~min}$ to form a water-in-oil-in-water (w/o/w) multiple emulsion. The emulsion was stirred to remove the methylene chloride above $2 \mathrm{~h}$. The PLGA-heparin nanoparticles were recovered by ultracentrifugation $(23,000 \mathrm{rpm}$ for $20 \mathrm{~min}$, Beckman, USA), washed 3 times to remove PVA, and then lyophilized to obtain a dry powder.

2.2. Characteristics of PLGA-Heparin Nanoparticles. A Submicro Laser Defractometer (PCS Brookhaven Co. USA, BI9000AT Correlator, BI200SM Photometer) was used to assess the size distribution. The particle morphology was observed by scanning electron microscopy. Freeze-dried nanoparticles were spread on the sticky surface of an aluminum stub. The nanoparticle surface was coated with gold before being viewed under the microscope.

The amount of heparin entrapped within PLGA nanoparticles was determined by extracting the heparin using distilled water after dissolving the PLGA with a chloroform solution. Heparin concentration in the aqueous solution was assayed by spectrophotometer.

In vitro heparin release from the nanoparticles was performed in distilled water at $37^{\circ} \mathrm{C}$ utilizing double-chamber diffusion cell. A Millipore hydrophilic polyvinylidene fluoride membrane was placed between the two chambers. The receiver buffer was replaced with fresh distilled water at predetermined intervals. Heparin concentration in the aqueous solution was assayed by spectrophotometer.

2.3. Mouse Hindlimb Ischemic Model. C57BL/6J mice at 4 weeks of age (15-18g body weight, from Laboratory Animals Center, Chinese Academy of Medical Sciences, China) were obtained. Hindlimb ischemic mouse model was surgically induced according to procedures described previously [19]. Briefly, mice underwent surgically induced unilateral hindlimb ischemia with ligation and excision of the femoral artery from its origin just above the inguinal ligament to its bifurcation at the origin of the saphenous and popliteal arteries under general anesthesia. The abovementioned arteries from the opposite limb were not ligated and were used as an internal control to calculate perfusion recovery.
The extent of necrosis, if any, in ischemic hindlimb was recorded once between days 3 and 5 after surgery. The scores of necrosis were described as follows: grade 0 , no necrosis in ischemic limb; grade I, necrosis limited to toes; grade II, necrosis extending to dorsum pedis; grade III, necrosis extending to curs; grade IV, necrosis extending to thigh.

Totally, 40 hindlimb ischemic mice were assigned to two groups: PLGA-heparin group $(n=20)$ and control group $(n=20)$. PLGA heparin $(300 \mu \mathrm{L}$ was equivalent to $6 \mathrm{IU}$ heparin; this dosage of heparin was equivalent to $300 \mathrm{IU} / \mathrm{kg}$ in clinic) was intramuscular injected into the ischemic limb (6 points) of each mouse right after the femoral artery was ligated and excised. Same amount of saline was intramuscular injected into the ischemic limb as we did in treatment group.

Blood flow recovery was monitored with Laser Doppler Perfusion Image (LDPI, PERIMED, Stockholm, Sweden). Briefly, mice were anesthetized and placed on a heating pad to maintain a constant temperature. Hair was removed with an electric shaver followed by a depilatory cream. LDPI was used to estimate dermal blood flow in the calf and foot before, immediately after procedures, and on days $7,14,21$, and 28 after hindlimb ischemia. Ratio of the operated/nonoperated limb was calculated to minimize the potential differences between ambient temperature and lighting.

\subsection{Histological Examination, ELISA, and Western Blot}

2.4.1. Preparation of Tissue Samples. Mice were sacrificed after LDPI at day 28 after-operation, and the gastrocnemius and tibialis anterior muscles from the right and left sides were harvested and weighed. The distal part of the muscle was frozen and embedded for histology study, and the proximal part was used either to detect vascular endothelial growth factor (VEGF) level with enzyme-linked immunosorbent assay (ELISA) or to determine hepatocyte growth factor (HGF) expression with Western blot.

\subsubsection{Endothelial Phosphate Alkaline Staining for Capillary} Density. Immunohistochemistry was performed on muscle sections of the gastrocnemius and tibialis anterior. Capillary per muscle fiber ratio was determined by endothelial phosphate alkaline staining; blue dots indicated capillaries. Capillary density was measured by counting six random high-power (magnification $\times 200$ ) fields or a minimum of 200 fibers from each ischemic and nonischemic limb on an inverted light microscope.

2.4.3. Expression of VEGF and HGF. Available ELISA for VEGF was performed on homogenized muscle samples as the manufacturer's instructions (R\&D Systems, USA). Briefly, leg muscle harvested from sacrificed mice was snap frozen in liquid nitrogen and pulverized by using a prechilled mortar and pestle on dry ice. Approximately $100 \mathrm{mg}$ of each muscle was homogenized in $1.5 \mathrm{~mL}$ NP-40 lysis buffer containing protease inhibitors. Muscle homogenates were centrifuged at $13000 \mathrm{rpm}$. The surface lipid layer was removed, and 
the supernatant was saved at $-80^{\circ} \mathrm{C}$ for ELISA and protein assay. Protein concentration was measured by using a $1: 500$ dilution of the supernatant in the microtiter plate by microBCA protein assay (Pierce, Rockford, USA). Supernatant dilutions of $1: 6$ were used for the VEGF ELISA assays.

Protein samples were separated on SDS-polyacrylamide gels and then transferred to a polyvinylidene difluoride membranes which were treated with blocking buffer (5\% skim milk). An anti-HGF antibody (Santa Cruz Biotechnologies, CA) was used as the primary antibody followed by incubation with an anti-mouse horseradish peroxidaseconjugated secondary antibody. Immune reactivity was visualized using ECL plus system. Quantification of results was performed by densitometry (Image J, NIH, USA), comparing the density of identically sized areas (corresponding to immunoreactive bands) and results analyzed as total integrated density (arbitrary units).

2.5. Statistical Analysis. Results were expressed as mean \pm standard deviation (SD). For comparison of mean data on PLGA-heparin treatment versus control, at one time point (i.e., VEGF HGF expression, capillary density), statistical significance was evaluated by use of 2-tailed Student's paired $t$ test. To determine the perfusion ratio over time, a standard variance ANOVA repeated measure was performed. Probability values $<0.05$ were considered to be significant.

\section{Results}

3.1. Characteristics of PLGA-Heparin Nanoparticles. A scanning electron microphotograph of heparin nanoparticles showed that the nanoparticles were spherical and discrete particles without aggregation and that they were smooth in surface morphology (Figure 1(a)). The average diameter of the nanoparticles was $297 \mathrm{~nm}$ (Figure 1(b)).

The loading efficiency of heparin in the nanoparticles was $5.35 \%$. The heparin release from nanoparticles in vitro was $40 \%$ in day 1 and $85 \%$ in day 3 and sustained over 14 days (Figure 2).

\subsection{PLAG Heparin Improved Blood Flow Restoration and Pre-} vented Limb Necrosis. The recovery of perfusion in PLGAheparin group was similar to that of control group on day $7(0.500 \pm 0.084$ versus $0.485 \pm 0.095)$. However, perfusion ratio was significantly increased on day $14(0.623 \pm 0.077$ versus $0.536 \pm 0.086, P<0.05)$, day $21(0.684 \pm 0.068$ versus $0.588 \pm 0.082, P<0.01)$, and day $28(0.733 \pm 0.093$ versus $0.606 \pm 0.079, P<0.01$ ) (Figure 3 ).

Immunohistochemical staining showed that capillary density was richer in PLGA-heparin group compared to that of in control group (in gastrocnemius, $0.19 \pm 0.08$ versus $0.30 \pm 0.08, P<0.05$; in tibialis anterior $0.22 \pm 0.11$ versus $0.33 \pm 0.04, P<0.05)$. Capillary density was represented as capillaries per muscle fiber (Figure 4).

The results of necrotic scores were consistent with the blood flow recovery and capillary density. With more capillaries formation and more blood flow restoration, the limb necrosis was much less in PLGA-heparin treated group.

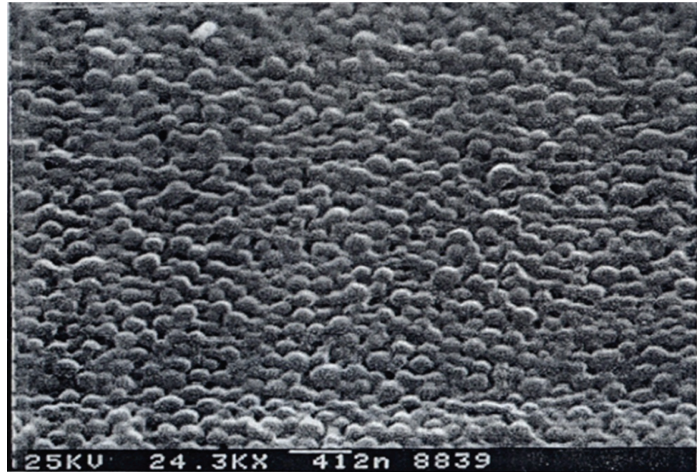

(a)

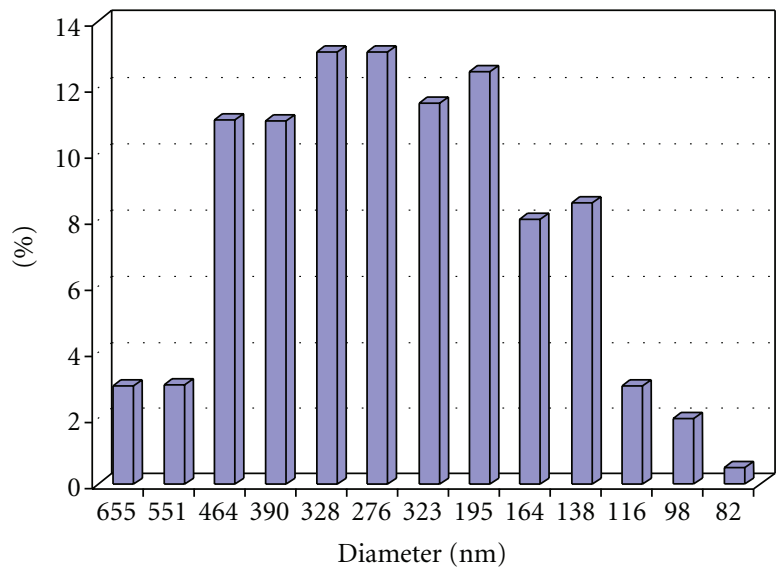

(b)

Figure 1: (a) A scanning electron microphotograph of heparin nanoparticles. (b) Size distribution of heparin nanoparticles.

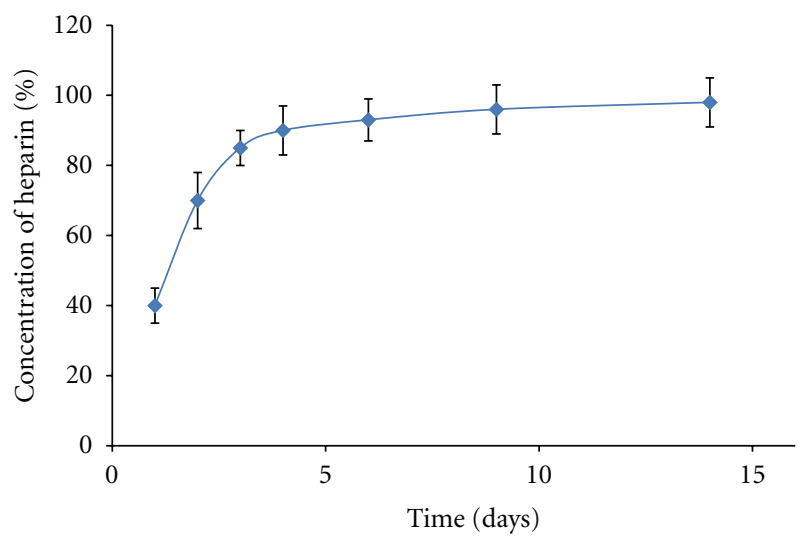

Figure 2: Cumulative release profile (\% of amount loaded) of heparin from nanoparticles.

Only 5\% mice showed grade I necrosis. However, in control group, $20 \%$ mice presented limb necrosis, and they were grade I (10.0\%), II (5.0\%), and III (5.0\%).

3.3. PLGA Heparin Upregulated the Expression of HGF. VEGF concentration was evaluated with ELISA. With ischemic injury stimulation, the VEGF level was increased 


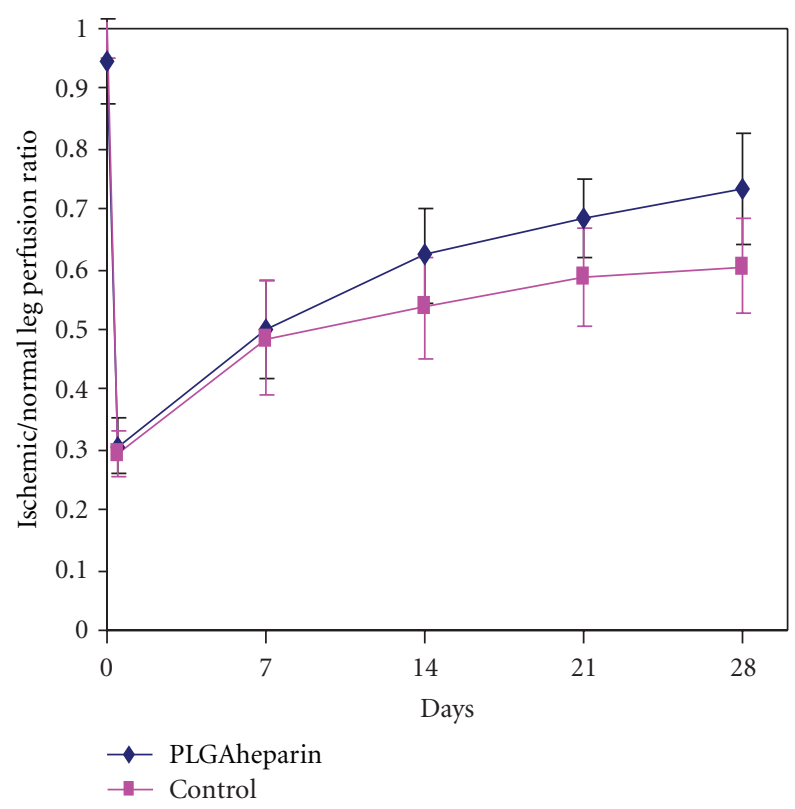

Figure 3: Perfusion recovery after surgical-induced hindlimb ischemia.

in both PLGA-heparin group and control group (20.6\% and $20.2 \%$ increased, respectively, $P>0.05$ ). There was no significant difference between these 2 groups.

However, western blot showed more expression of HGF in PLGA-heparin group than that in control group. Quantitative analysis with Image J showed the HGF/Tubulin ratio was significantly increased with PLGA-heparin versus control treatment, $0.43 \pm 0.02$ versus $0.20 \pm 0.06, P<0.05$, (integrated density) (Figure 5).

\section{Discussion}

Heparin is a typical anticoagulant and has been used to prevent and treat thromboembolic ischemic diseases such as coronary artery disease and peripheral arterial occlusion disease which imperil people's life or survive lower extremity limbs. However, a short half-life and the need for recurrent injection have limited its use for chronic ischemic disease. In this study, we attempt to use nanoparticle techniques to overcome this disadvantage and encapsulate heparin with PLGA to prolong its biological effect. To the best of our knowledge, this paper is the first to investigate the therapeutic efficiency of PLGA heparin in a preclinical hindlimb ischemic model. The major findings of our study were the following: (a) heparin could be encapsulated with PLGA and controlled release for at least 14 days; (b) PLGA heparin improved blood flow restoration with more capillaries formation and prevented limb necrosis; (c) the potential therapeutic angiogenesis of PLGA heparin might be due to interaction with growth factors.

According to our results, PLGA heparin nanoparticles formulated by double emulsion solvent evaporation technique were smooth and spherical in shape with a loading efficiency of 5.35\%. The average diameter of PLGA heparin was $297 \mathrm{~nm}$, with a range of $82 \mathrm{~nm}$ to $655 \mathrm{~nm}$. The heterogeneity in particular size might relate to the homogenization method. The concentration of PVA and the speed of homogenization had been reported to affect the particle size of PLGA nanoparticles $[20,21]$. The smaller particle size might have better potential effects of controlled release. The method of double-chamber diffusion was adopted for heparin release determination [22]. Result showed that heparin released from nanoparticles in vitro was $40 \%$ in day $1,85 \%$ in day 3 , and sustained over at least 14 days. Even though $90 \%$ of heparin was released from nanoparticle in the earlier 7 days, PLGA heparin did prolong the existence of heparin and made it possible to sustain the biological effects of heparin.

The controlled release of heparin from PLGA nanoparticles may offer the advantage of sustaining therapeutic effect in the target cells or tissues. A sustained drug release may be more important in the treatment of chronic disease such as critical limb ischemia and myocardial infarction. It also has the advantages that prolong the injection intermission for the convenience of clinic use and the improvement of patient compliance.

Our data showed that sustained heparin release from PLGA nanoparticles could be feasible and effective in therapeutic angiogenesis of mice in the setting of hindlimb ischemia. First of all, we detected the blood flow recovery with LDPI. Following surgical-induced hindlimb ischemia, the restoration of limb perfusion was spontaneous recovery over time, with one dosage of PLGA-heparin treatment; the restoration was accelerated. The differences demonstrated on day 14 following operation and lasted for 28 days. This finding was consistent with histological examination, with more capillaries formation in the PLGA-heparin treatment limbs. For clinical interest, less happening of necrosis on limbs confirmed the benefit of PLGA-heparin treatment.

We then sought to investigate the mechanisms of therapeutic angiogenesis of PLGA heparin. The vascular endothelial growth factor family is perhaps the most extensively studied angiogenic growth factor. VEGF is a key angiogenic factor that induces the proliferation, migration, and survival of endothelia cells and promotes angiogenesis [23]. We found an increase of VEGF expression during the recovery period after surgical-induced hindlimb ischemia. However, there was not distinct difference between the PLGA-heparin treatment and the saline control groups. That implied that there might be other mechanisms for promoting angiogenesis.

In this study, we found that PLGA heparin could upregulate the expression of HGF. HGF plays important roles in wound healing, tissue and organ regeneration, morphogenesis, and angiogenesis [24]. HGF and its specific receptor, c-met, have been expressed in the blood vessels, including endothelial cells and vascular smooth muscle cells. HGF had been confirmed as a novel angiogenic growth factor in models of hindlimb ischemia and myocardial infarction [25]. Moreover, clinical trials which are based on those bench works are on the way [26].

Taking all this information together, we conclude that it is a feasible and effective method to encapsulate the heparin 


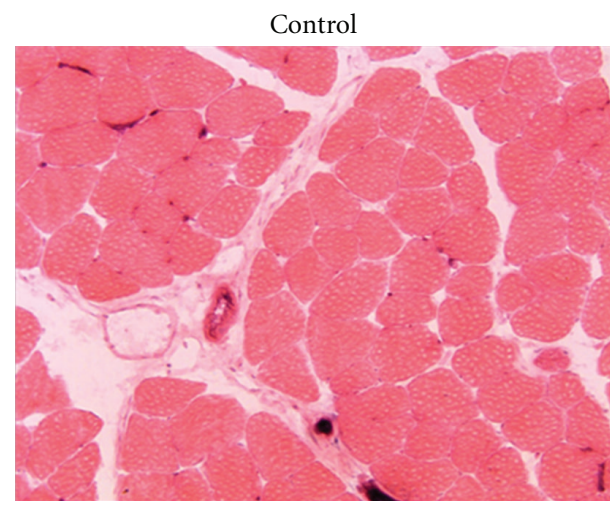

(a)

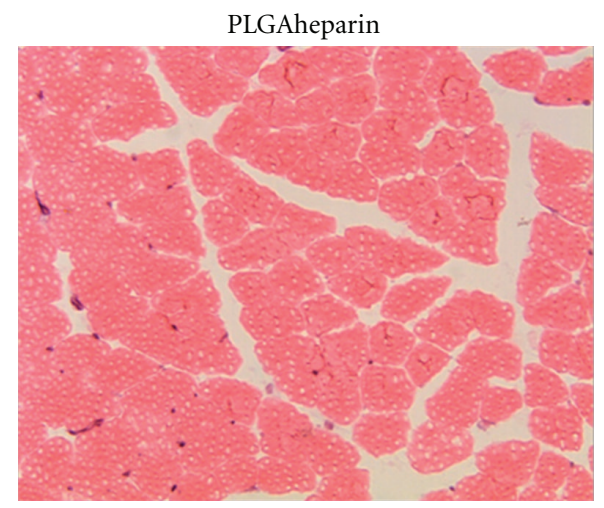

(b)

FIGURE 4: Endothelial phosphate alkaline staining for capillaries. The pictures represented staining of endothelial phosphate alkaline; blue dots indicated capillaries (original magnification $\times 200$ ).
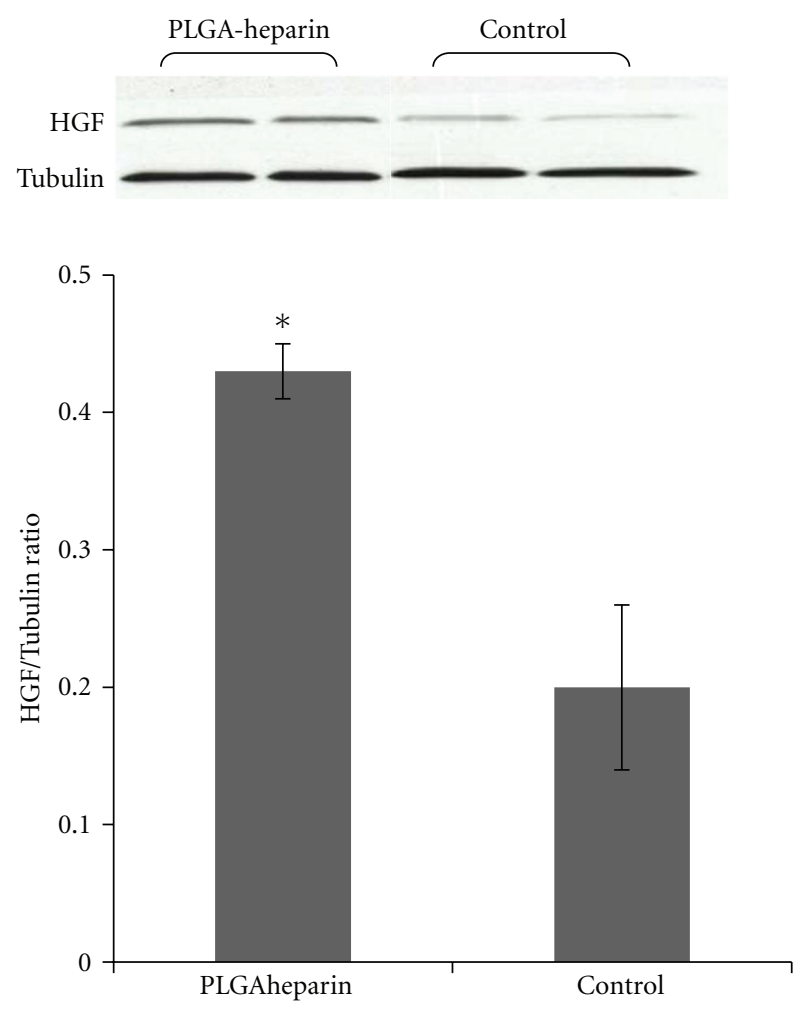

Figure 5: Western blot for HGF. Quantitative analysis with integrated density showed significantly more expression of HGF in PLGA-heparin group than that in control group.

into PLGA, and with controlled release of heparin, PLGAheparin can promote therapeutic angiogenesis in hindlimb ischemic mouse model. The potential mechanisms may be due to the upregulation of angiogenic factors.

\section{Acknowledgment}

This paper was supported by the National Natural Science Foundation of China (Grant no. 30772114).

\section{References}

[1] Y. He, Y. Jiang, J. Wang, L. Fan, X. Li, and F. B. Hu, "Prevalence of peripheral arterial disease and its association with smoking in a population-based study in Beijing, China," Journal of Vascular Surgery, vol. 44, no. 2, pp. 333-338, 2006.

[2] Y. H. Kusumanto, V. Van Weel, N. H. Mulder et al., "Treatment with intramuscular vascular endothelial growth factor gene compared with placebo for patients with diabetes mellitus and critical limb ischemia: a double-blind randomized trial," Human Gene Therapy, vol. 17, no. 6, pp. 683-691, 2006.

[3] B. Cristofaro, O. A. Stone, A. Caporali et al., "Neurotrophin-3 is a novel angiogenic factor capable of therapeutic neovascularization in a mouse model of limb ischemia," Arteriosclerosis, Thrombosis, and Vascular Biology, vol. 30, no. 6, pp. 11431150, 2010.

[4] H. Zhou, Y. H. Yang, N. O. Binmadi et al., "The hypoxiainducible factor-responsive proteins semaphorin $4 \mathrm{D}$ and vascular endothelial growth factor promote tumor growth and angiogenesis in oral squamous cell carcinoma," Experimental Cell Research. In press.

[5] M. Sobel and R. Verhaeghe, "Antithrombotic therapy for peripheral artery occlusive disease: American College of Chest Physicians evidence-based clinical practice guidelines (8th edition)," Chest, vol. 133, no. 6, pp. 815S-843S, 2008.

[6] C. Kearon, S. R. Kahn, G. Agnelli, S. Goldhaber, G. E. Raskob, and A. J. Comerota, "Antithrombotic therapy for venous thromboembolic disease: American College of Chest Physicians evidence-based clinical practice guidelines (8th edition)," Chest, vol. 133, no. 6, pp. 454S-545S, 2008.

[7] L. Norgren, W. R. Hiatt, J. A. Dormandy, M. R. Nehler, K. A. Harris, and F. G. R. Fowkes, "Inter-Society consensus for the management of peripheral arterial disease (TASC II)," European Journal of Vascular and Endovascular Surgery, vol. 33, pp. S32-S53, 2007.

[8] D. A. Lane, "Heparin binding and neutralizing protein," in Heparin: Chemical and Biological Properties, Clinical Applications, D. A. Lane and U. Lindahl, Eds., pp. 363-391, CRC Press, Boca Raton, Fla, USA, 1989.

[9] R. D. Rosenberg and L. Lam, "Correlation between structure and function of heparin," Proceedings of the National Academy of Sciences of the United States of America, vol. 76, no. 3, pp. 1218-1222, 1979. 
[10] J. Hirsh, T. E. Warkentin, S. G. Shaugnessy et al., "Heparin and low-molecular-weight heparin: mechanisms of action, pharmacokinetics, dosing considerations, monitoring, efficacy and safety," Chest, vol. 119, supplement, pp. 64S-94S, 2001.

[11] M. Stern, K. Ulrich, D. M. Geddes, and E. W. F. W. Alton, "Poly (D, L-lactide-co-glycolide)/DNA microspheres to facilitate prolonged transgene expression in airway epithelium in vitro, ex vivo and in vivo," Gene Therapy, vol. 10, no. 16, pp. 12821288, 2003.

[12] S. W. Kang, H. W. Lim, S. W. Seo, O. Jeon, M. Lee, and B. S. Kim, "Nanosphere-mediated delivery of vascular endothelial growth factor gene for therapeutic angiogenesis in mouse ischemic limbs," Biomaterials, vol. 29, no. 8, pp. 1109-1117, 2008.

[13] O. Jeon, S. W. Kang, H. W. Lim, J. Hyung Chung, and B. S. Kim, "Long-term and zero-order release of basic fibroblast growth factor from heparin-conjugated poly(L-lactide-coglycolide) nanospheres and fibrin gel," Biomaterials, vol. 27, no. 8, pp. 1598-1607, 2006.

[14] J. Yang, C. Song, Y. Li et al., "Polymeric Particles with therapeutic gene for gene therapy: preparation and in vivo gene transfer study," BioMedical Engineering, vol. 22, no. 3, pp. 438-442, 2005.

[15] Y. Lemmouchi, E. Schacht, P. Kageruka et al., "Biodegradable polyesters for controlled release of trypanocidal drugs: in vitro and in vivo studies," Biomaterials, vol. 19, no. 20, pp. 18271837, 1998.

[16] R. A. Jain, "The manufacturing techniques of various drug loaded biodegradable poly(lactide-co-glycolide) (PLGA) devices," Biomaterials, vol. 21, no. 23, pp. 2475-2490, 2000.

[17] X. Liu, J. Yang, C. Zhou et al., "Constructing hybrid films of conjugated oligomers and gold nanoparticles for efficient photoeletronic properties," Physical Chemistry Chemical Physics, vol. 13, no. 6, pp. 1984-1989, 2011.

[18] A. Lamprecht, N. Ubrich, M. Hombreiro Pérez, C. M. Lehr, M. Hoffman, and P. Maincent, "Biodegradable monodispersed nanoparticles prepared by pressure homogenizationemulsification," International Journal of Pharmaceutics, vol. 184, no. 1, pp. 97-105, 1999.

[19] T. Couffinhal, M. Silver, L. P. Zheng, M. Kearney, B. Witzenbichler, and J. M. Isner, "Mouse model of angiogenesis," American Journal of Pathology, vol. 152, no. 6, pp. 1667-1679, 1998.

[20] D. R. Kalaria, G. Sharma, V. Beniwal, and M. N. V. Ravi Kumar, "Design of biodegradable nanoparticles for oral delivery of doxorubicin: in vivo pharmacokinetics and toxicity studies in rats," Pharmaceutical Research, vol. 26, no. 3, pp. 492-501, 2009.

[21] J. Sameni, N. I. Bukhari, N. A. Azlan, T. Julianto, and A. B. A. Majeed, "The effect of preparation parameters on the size and morphology of PLGA-based nanoparticles," in Proceedings of the IEEE Symposium on Industrial Electronics and Applications (ISIEA '09), pp. 700-704, Kuala Lumpur, Malaysia, October 2009.

[22] J. Lv, Y. Zhao, G. Li et al., "Aggregation-enhanced emission in gold nanoparticles protected by tetradentate perylene derivative," Langmuir, vol. 25, no. 19, pp. 11351-11357, 2009.

[23] T. T. Rissanen, I. Vajanto, M. O. Hiltunen et al., "Expression of vascular endothelial growth factor and vascular endothelial growth factor receptor-2 (KDR/Fik-1) in ischemic skeletal muscle and its regeneration," American Journal of Pathology, vol. 160, no. 4, pp. 1393-1403, 2002.
[24] R. Zarnegar and G. K. Michalopoulos, "The many faces of hepatocyte growth factor: from hepatopoiesis to hematopoiesis," Journal of Cell Biology, vol. 129, no. 5, pp. 1177-1180, 1995.

[25] R. Madonna, C. Cevik, M. Nasser et al., "Hepatocyte growth factor: molecular biomarker and player in cardioprotection and cardiovascular regeneration," Thrombosis and Haemostasis, vol. 107, no. 4, pp. 656-661, 2012.

[26] R. Morishita, H. Makino, M. Aoki et al., "Phase I/IIa clinical trial of therapeutic angiogenesis using hepatocyte growth factor gene transfer to treat critical limb ischemia," Arteriosclerosis, Thrombosis, and Vascular Biology, vol. 31, no. 3, pp. 713-720, 2011. 

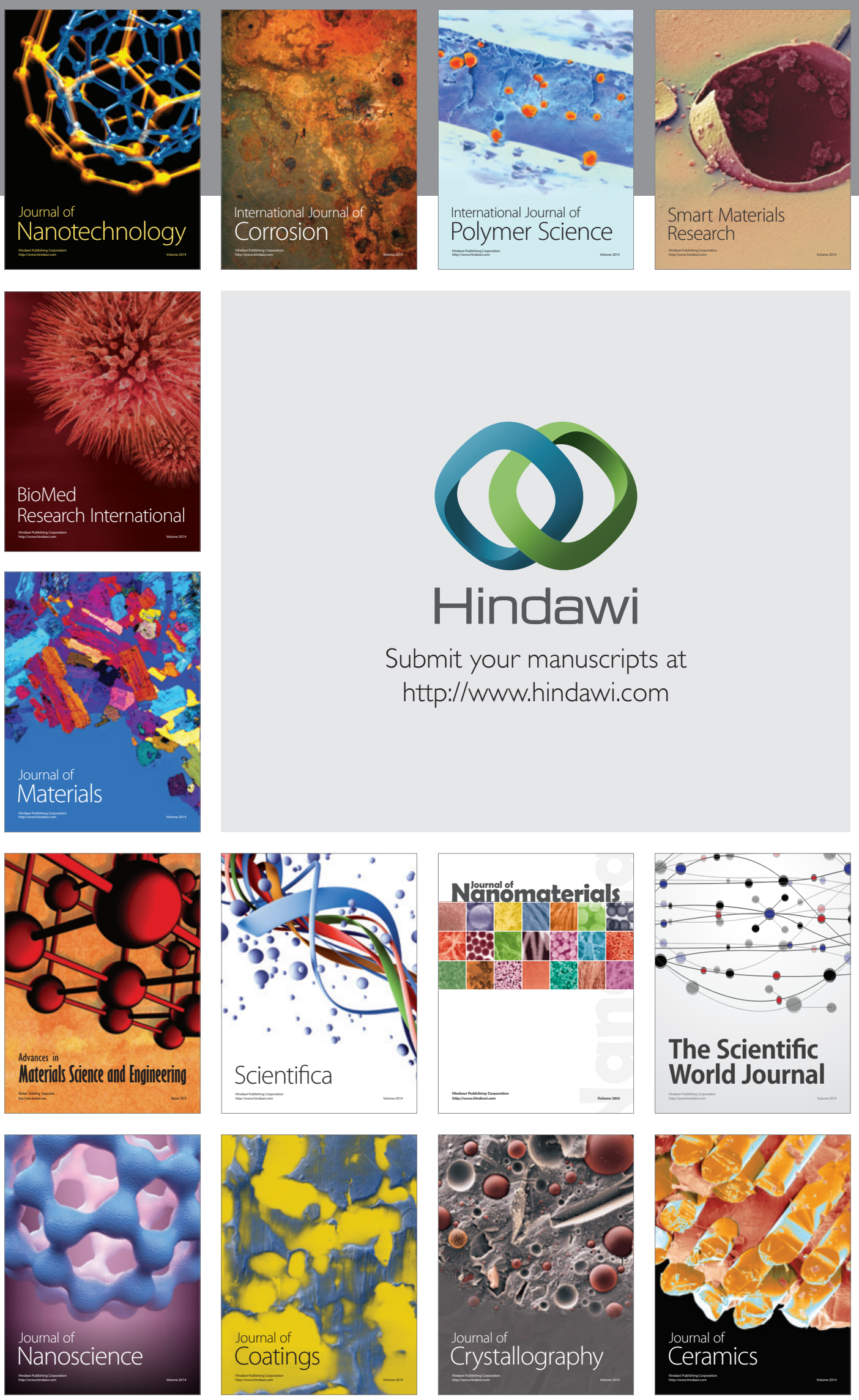

The Scientific World Journal

Submit your manuscripts at

http://www.hindawi.com

\section{World Journal}

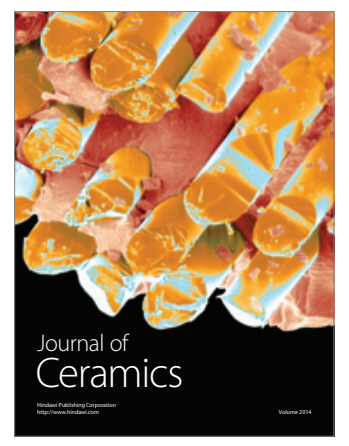

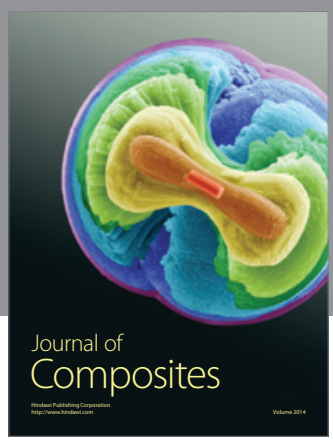
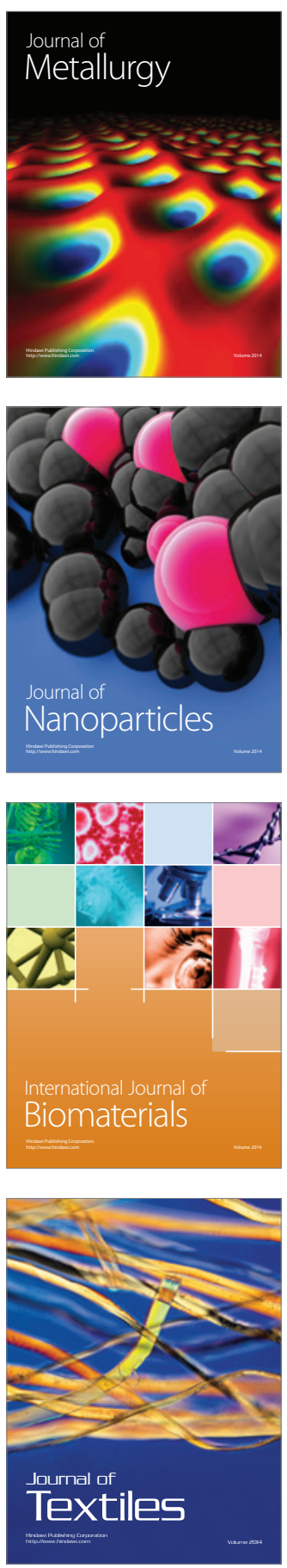